\title{
Des communautés de pratique aux réseaux de pratique: une étude de l'évolution d'une communauté des startups de la ville de Belo Horizonte
}

\author{
TAtiane Barleto Canizela Guimarães ${ }^{12}$ \\ LUCIANA CASTRO Gonçalves ${ }^{3}$ \\ GLÁUCIA MARIA VASCONCELlos VALE ${ }^{4}$ \\ ${ }^{1}$ Centro Universitário de Belo horizonte (UniBh), Belo Horizonte - MG, Brésil \\ ${ }^{2}$ Centro Universitário newton Paiva, Belo Horizonte - MG, Brésil \\ 3 UnIVERSité PARIS-EST / ESIEE PARIS, INSTITUt de RECHERCHE EN GESTION, PARIS, FRANCE \\ ${ }^{4}$ Pontifícia Universidade Católica de Minas Gerais (PuC Minas), Belo Horizonte - MG, Brésil
}

\section{Résumé}

Cette recherche explore le lien la littérature de communauté de pratique et réseau de pratique de façon évolutive dans un contexte d'innovation. L'analyse des échanges de connaissances et de ressources de la communauté d'entrepreneurs de San Pedro Valley, localisé dans la ville de Belo Horizonte, permet de montrer ses évolutions selon trois processus de participation/réification distincts qui marquent sa légitimité dans l'écosystème d'innovation de la ville. D'un groupe informel, émerge une communauté de pratique inter-organisationnelle que devient progressivement un réseau de pratique selon un continuum où la pratique co-évolue avec l'écosystème que devienne plus fort.

Mots-clés: Communautés de pratique. Réseaux de Pratique. Dynamique d'innovation. Relations inter-organisationnelles.

De comunidades de prática a redes de práticas: um estudo da evolução de uma comunidade de startups da cidade de Belo Horizonte

\section{Resumo}

Esta pesquisa explora a relação entre as literaturas de comunidade de prática e redes de prática de maneira evolutiva em um contexto de inovação. A análise das trocas de conhecimentos e de recursos da comunidade de empreendedores de San Pedro Valley, em Belo Horizonte, permite mostrar sua evolução em três processos de participação/reificação distintos que marcam a legitimidade crescente no ecossistema de inovação da cidade. De um grupo informal emerge uma comunidade de pratique interorganização, que se transforma progressivamente em uma rede de pratica de acordo com um continuum, no qual a prática e o ecossistema que se fortalecem e coevoluem conjuntamente.

Palavras-chave: Comunidade de prática. Redes de prática. Dinâmicas de inovação. Relações interorganizacionais.

\section{From community of practice to network of practice: a study of the evolution of a startup community in the city of} Belo Horizonte

\section{Abstract}

This research explores the relationship from the literature of community of practice and network of practice in an evolutionary process in a context of innovation. An analysis of the knowledge and resources exchanges of the San Pedro Valley entrepreneur's network, in Belo Horizonte, Brazil, shows its evolution in three distinct participation/reification processes that mark the growing legitimacy of the city's innovation ecosystem. From an informal group emerges an inter-organizational community of practice, which is progressively transformed into a network of practice according to a continuum, where the practice co evolves together with the ecosystem that is reinforced.

Keywords: Community of practice. Networks of practice. Dynamics of innovation. Interorganizational relationships. 


\section{INTRODUCTION}

La coopération au sein des communautés est devenue un thème courant dans les recherches sur l'innovation. Les communautés de pratique (CoP) apparaissent comme une forme particulière de réseau informel d'individus partageant le même intérêt ou une passion autour d'un thème spécifique et comportant une forte dimension identitaire (WENGER, MCDERMOTT et SNYDER, 2002). Leurs spécificités de fonctionnement favorisent les échanges d'informations, de connaissances et de ressources permettent ainsi de répondre aux besoins et aux objectifs individuels et collectifs tout en confiance (WENGER, 1998; BROWN et DUGUID, 1991; WENGER, 1998; WENGER, MCDERMOTT et SNYDER, 2002; COHENDET, GRANDADAM, SIMON et al. 2014; GHERARDI, 2015).

Devenir membre d'une CoP est un processus qui s'inscrit dans le temps, à travers l'engagement mutuel de leurs pratiques, partagées au sein d'un répertoire commun (BROWN et DUGUID, 1991; WENGER, 1998). L'action de quelqu'un ne devient significative que par rapport aux actions des autres. L'innovation apparaît donc à partir de cette interaction entre membres.

Dans le contexte actuel, l'innovation constitue un facteur clé de succès des entreprises et un moteur de l'économie fondée sur des changements continus et où les technologies avancent significativement pour faire face à la concurrence (JENSEN, JOHNSON, LORENZ et al., 2007; LOILIER et TELLIER, 2013). La littérature sur les CoP fait part d'une reconnaissance partagée du potentiel de son potentiel à favoriser l'apprentissage collectif au sein des entreprises (MACDERMOT, 2000; PATTISSON et PREECE, 2014) et des villes (COHENDET et SIMON, 2010; COHENDET, GRANDADAM, SIMON et al., 2014).

Bien que initialement fondés principalement sur des organisations individuelles, les études les plus récents sur la coopération ont mis l'accent sur les pratiques et les processus de collaboration inter-organisationnelles (par exemple: BERENDS, VAN BURG et VAN RAAIJ, 2011; LEVINA et VAAST, 2005; HISLOP, 2016; FIGUEIREDO et PIANA, 2016). De ce point de vue, les CoP interorganisationnelles présentent un intérêt particulier dans les écosystèmes car elles sont considérées comme « un moyen efficace pour les organisations de manière à gérer les problèmes non structurés ou non construits et de partager leurs connaissances au-delà des limites structurelles traditionnelles » (LESSER et STORCK, 2001, p. 832; AGARWAL et AGARWAL, 2017).

Dans cette ligne, Fichter et Beucker (2012), examinent comment les communautés d'innovation peuvent être activement soutenues et gérées en orientant ou influençant leur configuration ou leur offrent de structures. C'est auteurs affirment qu'il n'y a pas de recette standard pour leur construction et gestion.

Selon Agterberg, Bart, Marleen et al. (2010), au niveau des relations inter-organisationnelles, les CoP auront tendance à se disperser géographiquement et devenir des réseaux de pratique (des Network of Practice ou NoP). À l'échelle d'une ville, ces réseaux sont des générateurs d'apprentissage collectif contribuant au développement de l'intelligence territoriale (JOYAL, 2008). Cependant, encore trop peu de travaux s'intéressent à l'analyse des rapports de réciprocité évolutive entre la pratique, la communauté, le réseau et l'espace qui la fait vivre. Cette limite risque de donner une vision statique des CoP alors que la littérature souligne que ces unités sont dynamiques et peuvent dépasser les frontières des organisations. Ainsi, la problématique de cette recherche est formulée de la façon suivante : "Comment les CoP évoluent et contribuent aux contextes inter-organisationnels étendus dans un territoire où l'innovation prime ? »

La notion de NoP semble alors pertinente pour étudier les CoP inter-organisationnelles en ce qu'elles intègrent une dimension géographique. Notre intérêt, dans ce travail porte sur l'analyse d'une communauté d'entrepreneurs formée par différentes startups qui se situent dans une même région géographique. Le point de départ de notre recherche est la dynamique sociale d'une communauté de pratique inter-organisationnelle. Notre recherche insiste sur le développement de CoP inter-organisationnelles constituées de membres très diversifiés et provenant de plusieurs organisations (TEIGLAND, 2000; MOINGEON, QUÉLIN, DALSACE et al., 2006 ; LETOURNEAU et al., 2011; COHENDET, 2014) de façon évolutive dans un contexte d'innovation. Pour ce faire, nous analysons l'évolution d'une communauté d'entrepreneurs des startups à Belo Horizonte, depuis son émergence jusqu'à nos jours, détaillant l'ensemble de transformations ayant eu lieu dans ce réseau et plus généralement dans la ville où elle se situe.

L'objectif de ce travail est d'analyser si quand une CoP atteint une masse critique de participants, elle devient un NoP et comment cette évolution engendre des transformations au niveau de sa pratique et de son fonctionnement dans l'espace régional qui l'accueille. 


\section{Des CoP au NoP : Quelle Application en Contexte d'innovation dans les Territoires?}

Dans le contexte des CoP et des NoP, la pratique fonde le liant entre ses membres, sachant que le dernier incorpore les dimensions sociales et identitaires du collectif d'individus (LAVE et WENGER, 1991), c'est qu'amène à considérer l'apprentissage selon une approche sociale et située (ibid.). Nous présentons les principes qui régissent cette dynamique.

\section{Les CoP et les NoP : état de l'art}

Le terme communauté de connaissance est un terme générique utilisé pour caractériser les différents types de groupes formels et informels visant à créer et à maintenir les connaissances dans les organisations.

La notion de CoP a été créée par Lave et Wenger (1991) au moment où ils ont identifié des groupes d'individus engagés dans la même pratique, qui si communiquaient de façon régulière au sujet de leurs activités. Ces auteurs considèrent les CoP comme le vecteur d'apprentissage porté par la pratique de membres. Le but de leurs membres est de développer leurs compétences dans la pratique, à travers la construction, l'échange et le partage des ressources (WENGER, 1998). Ainsi, « les communautés de pratique sont des groupes d'individus partageant le même intérêt, le même ensemble de problèmes, ou une passion autour d'un thème spécifique et qui approfondissent leur connaissance et leur expertise en interagissant régulièrement» (WENGER, MCDERMOTT et SNYDER, 2002, p. 4).

Les CoP sont donc une forme particulière de réseau social (DAMERON et JOSSERAND, 2009). La pratique apporte le socle de la structure de la CoP à travers son domaine de savoir (l'objet commun qui incite ses membres à se rassembler), son fonctionnement (l'engagement mutuel, la passion commune, les « rituels » de rencontres) et sa capacité de produire la pratique (sur la base d'un référentiel commun fondé sur des langages propres, des leçons d'apprentissage, des histoires et des sensibilités partagées, des artefacts, des objets spécifiques à la communauté) (WENGER, 1998).

De nombreux travaux ont été publiés par d'autres auteurs en contexte organisationnel, au sein d'une même organisation ayant un contexte stable (DIBIAGGIO et FERRARY, 1999; COHENDET, CREPLET et DUPOUET, 2003; JOSSERAND, 2004) et dans des contextes ambigus et évolutifs (par ex. VAAST, 2004; CASTRO GONÇALVES, 2015). Cependant, les analyses n'abordent pas assez le contexte inter-organisationnel de ces relations (COHENDET, GRANDADAM, SIMON et al., 2014), ni la nature des dynamiques sociales, ni les tensions qui peuvent émerger en présence de relations de concurrence et évolutifs (LEVERING, LIGTHART, NOORDERHAVEN et al. 2013).

Pourtant, nous considérons que ces aspects évolutifs et concurrentiels sont extrêmement importants lorsque les CoP se construisent dans des contextes émergents et dynamiques.

Dans cette lignée, une nouvelle vague de travaux a développé la notion de NoP. Brown et Duguid (1991) utilisent le terme pour décrire des relations entre les membres qui sont clairement plus lâches que celles d'une CoP. Agterberg, Bart, Marleen et al. (2010) défendent le fait que la notion de NoP aurait été proposée pour introduire le point de vue de la vision basée sur les ressources (RBV) pour l'analyse du partage de la connaissance dispersée géographiquement (GOGLIO-PRIMARD, 2014).

Au sein des NoPs, des réunions informelles et des groupes de travail peuvent être créés pour apprendre, partager des connaissances et offrir aux acteurs un espace de comparaison de leurs pratiques. Même si ils ne sont pas nommées comme telles, ils présentent des nombreuses caractéristiques propres aux CoP (WENGER, 1998).

Les NoPs aussi bien que les CoPs se réfèrent à des groupes de personnes qui se livrent à des pratiques communes. Mais, les NoPs ne sont pas nécessairement co-localisées et sont composées de participants présents sur une grande échelle.

C'est l'encastrement des connaissances dans le réseau et sa pratique et l'encastrement relationnel ou structurel des membres qui structurent les NoP. Agterberg, Bart, Marleen et al. (2010) utilisent le terme de "embeddedness" pour évoquer les trois niveaux d'encastrement nécessaires pour un partage efficace des connaissances entre les unités dispersées:

- l'encastrement de la pratique : souligne que plus la connaissance est partagée dans le réseau plus elle sera perçue comme pertinente par rapport aux pratiques locales ;

- l'encastrement social : met l'accent sur le fait que plus les membres sont conscients de qui sait quoi dans le réseau, plus le réseau est caractérisé par de forts liens sociaux ;

- l'encastrement organisationnel : affirme que plus les organisations sont impliquées dans le réseau, plus elles sont en mesure d'apprendre de la connaissance qui est partagée. 
Des communautés de pratique aux réseaux de pratique: une étude de l'évolution d'une communauté des startups de la ville de Belo Horizonte
Tatiane Barleto Canizela Guimarães | Luciana Castro Gonçalves Gláucia Maria Vasconcellos Vale

Le niveau de partage des connaissances et d'autres types de sources sera d'autant plus important si les différents degrés d'encastrement sont élevés. Alors qu'au sein des CoP les connaissances font l'objet d'échanges au niveau interindividuel, au sein des NoP, les ressources deviennent davantage l'objet de ces échanges au niveau interorganisationnel.

Wenger (2000, p. 193) affirme que les communautés et les réseaux sont des structures distinctes : " plutôt que de mettre en contraste une communauté et un réseau, il est plus utile de penser la communauté et le réseau comme deux types de processus structurants $"$.

La CoP met l'accent sur l'identité tandis que le NoP met l'accent sur la connectivité. Habituellement, les deux coexistent. Certes, les CoP sont des NoP dans le sens où elles impliquent des connexions entre membres. Mais, il y a aussi l'identification avec un domaine et l'engagement à un partenariat d'apprentissage, qui ne sont pas nécessairement présents dans les NoPs.

Le tableau 1 ci-dessous récapitule les principes des dynamiques à l'œuvre dans les CoP et les NoP relevés dans la littérature.

Tableau 1

Les principes des dynamiques à l'œuvre dans les CoP et les NoP

\begin{tabular}{|l|l|l|}
\hline \multicolumn{1}{|c|}{ Principes } & \multicolumn{1}{c|}{ CoP } & \multicolumn{1}{c|}{ NoP } \\
\hline Finalité de la pratique & $\begin{array}{l}\text { Partage de connaissances en vue de la } \\
\text { résolution de problèmes ou partage d'une } \\
\text { passion }\end{array}$ & $\begin{array}{l}\text { Partage de ressources } \\
\text { technologiques, physiques et } \\
\text { managériales }\end{array}$ \\
\hline Fréquence des rencontres & Régulières et co-localisées & $\begin{array}{l}\text { Peu régulières et dispersées } \\
\text { géographiquement }\end{array}$ \\
\hline Principe communautaire & Entreprise commune & Encastrement dans la pratique \\
\hline Dynamique sociale & Engagement mutuel des membres & Encastrement social \\
\hline Capacité de reproduire la pratique & Sur la base d'un répertoire partagé & Encastrement organisationnel \\
\hline
\end{tabular}

Source : Adapté de Wenger (1998); Agterberg, Bart, Marleen et al. (2010).

L'intérêt des concepts de CoP et de NoP pour analyser les dynamiques communautaires se repose sur la proposition d'un cadre analytique propice pour étudier le lien entre les phénomènes interindividuels (niveaux micro-organisationnels des acteurs) et la création de connaissances inter-organisationnelles en jeu (COHENDET, GRANDADAM, SIMON et al., 2003). Nous nous intéressons à présent à une mise en perspective évolutive de ces dynamiques communautaires.

\section{Des CoPs aux NoPs : pour une vision évolutive}

L'observation de l'intégration de nouveaux membres au sein des CoP selon les différents niveaux d'appartenance - au noyau dur, niveau des actifs, des occasionnels et de la périphérie - offre une première perceptive évolutive de ces entités. Les membres d'une communauté s'auto-sélectionnent et leur participation peut évoluer en passant d'un niveau à un autre (WENGER et SNYDER, 2000). Cette utilisation d'appartenance multiple est cependant insuffisante pour étudier l'évolution des CoP dans le temps en ce qu'elle vise plutôt à montrer comment les individus créent de nouveaux liens de façon à permettre la coordination.

Ainsi McDermott (2000), propose de voir le cycle de vie d'une CoP en cinq phases d'un processus délibéré : planifier, lancer, croître, soutenir, arrêter. Cette vision omet cependant le caractère spontané de ces organisations.

Wenger, Mcdermott e Snyder (2002) précisent par la suite une dynamique évolutive plus large structurée également en cinq étapes : potentialité, regroupement, maturation, organisation, transformation.

Ces modèles présentent cependant certaines limites. II existe en effet une distance entre la richesse conceptuelle initiale proposée par Wenger (1998) et la mise en évidence de « cycles ». Gongla et Rizzuto (2001) offrent une perspective à cette limite en proposant d'étudier leur évolution par un enchaînement de phases qui résultent plus de l'influence de contingences internes et environnementales que d'un processus continu et «préprogrammé» (DAMERON et JOSSERAND, 2007). 
Dans cette optique, la dualité participation/réification (WENGER, 1998) nous apparaît comme une réponse possible pour étudier l'évolution des CoPs aux NoPs.

Wenger $(1998,2005)$ propose la dualité de la participation / réification pour comprendre les processus d'apprentissage, de production de sens et d'identité.

La notion de participation est utilisée " pour décrire l'expérience sociale de vie dans le monde, d'appartenance à des communautés sociales et d'engagement dynamique dans des projets collectifs " (WENGER, 2005, p. 62). Est un processus complexe, personnel et social, qui engage l'individu dans sa totalité et qui s'accompagne de plusieurs gestes tels que faire, parler, ressentir et appartenir. La participation devient une source d'identité, une « identité de participation ».

Par ailleurs, la notion de réification décrit «le processus qui consiste à donner une forme à notre expérience en créant des objets qui la cristallisent en « chose» » (WENGER, 2005, p. 64). Elle créée des points de convergence autour de la négociation de sens, offre une forme adaptée à l'interprétation, pour résoudre un conflit et en tant qu'outil. La réification donne une forme concrète à l'expérience des membres par plusieurs formes : une formule abstraite, un petit logo, une note de service, un regard révélateur.

L'intérêt dans cette recherche consiste à étudier l'évolution de cette " tension » dynamique présente dans des CoPs en contexte d'innovations en partant du principe que ces entités peuvent devenir des NoP. Nous considérons que cette analyse peut permettre d'analyser le passage d'un groupe communautaire contré à un groupe qui devient plus large et est fondé sur des relations interorganisationnelles dispersées géographiquement.

Au-delà de l'analyse de l'évolution du réseau d'individus et de ses caractéristiques dans le contexte spécifique de l'innovation, il serait intéressant de nous pencher également sur les effets que cette évolution peut produire dans l'espace géographique qui l'accueille. Le lien entre innovation et territoire sont en effet de plus en plus présents dans la littérature.

Les contextes d'innovation comportent des différents attributs dans la littérature. Réfléchir en termes d'innovation implique d'identifier une dimension liée à la nouveauté dans la configuration des rapports sociaux mais également, d'enquêter sur l'inscription durable ou non de cette nouveauté dans le monde social.

Tandis que dans les approches classiques des stratégies des CoP et des NoP négligent la compétition, cette caractéristique présente dans le relations interorganisationnelles entraine des avantages mutualisés pour les membres tandis qu'émerge l'idée d'une dialectique des relations (l'existence de contradictions, de forces opposées) qui change la dynamique sociale des CoP. C'est ainsi que les dynamiques sociales sont soumises à des tensions (autonomie versus contrôle, coopération versus compétition, etc.) et évolutions.

La participation trouve une valeur pour les interactions des membres impliqués, au-delà de leur travail, car ils obtiennent également une satisfaction personnelle lorsqu'ils partagent des choses avec des personnes qui comprennent leurs préoccupations ou sont confrontées à des problèmes similaires. Les relations reposent sur une reconnaissance informelle des membres. Les membres sont des individus appartenant à des organisations différentes qui décident de collaborer (MOINGEON, QUÉLIN, DALSACE et al., 2006).

La littérature sur les CoP et NoP est riche mais présente encore des lacunes conceptuelles permettant une opérationnalisation aisée dans des contextes interorganisationnels où l'innovation prime au sein d'un territoire. Afin d'apporter des contributions à ce limites nous analysons le cas d'une communauté d'entrepreneur de startups dans la ville de Belo Horizonte.

\section{La Méthodologie de la Recherche}

Pour répondre à cette problématique, nous avons privilégié une méthodologie qualitative, de nature exploratoire (GHAURI et GRONHAUG, 2005), recourant à une étude de cas de type longitudinal exploratoire (YIN, 2002).

Dans cette recherche nous avons eu l'opportunité d'étudier la communauté appelée San Pedro Valley (CoP de SPV), créée en 2011 de façon spontanée et émergente par un groupe d'entrepreneurs de startups innovants.

Avant de présenter les instruments de collecte de données mobilisés, nous justifions le choix de notre terrain de recherche tout en exposant sa représentativité théorique. 


\section{Le terrain de recherche : le réseau d'entrepreneurs San Pedro Valley}

Selon les données de l'Association Brésilienne des Startups dans l'année de 2015 le secteur de startup a augmenté 38\% dans l'état de Minas Gerais et 30\% dans le Brésil. Selon les données publiées par l'institution, le pays possédé plus de 4200 startups, desquelles plus de 590 situés dans l'état de Minas Gerais, ce qui rend la ville comme une référence pour le secteur.

Selon les données de la ABStartup (2017), la ville de Belo Horizonte présente 418 startups, la major densité d’entreprises de TI du Brésil. En 2015 il y avait environ 331 entreprises de technologies par chaque 100 mil habitant alors que seule l'état de Minas Gerais présentée 111 entreprises de technologies para chaque 100 mil habitant c'est qui lui place dans une position importante vis-à-vis les autres villes de l'État et du pays.

Dans ce contexte propice à l'innovation a émergé spontanément la communauté de SPV en 2011 dans le but de favoriser le partage des connaissances entre les startups situées dans le quartier "São Pedro ». Les entrepreneurs souffraient du manque de ressources financières, technologiques et structurelles et d'une difficulté d'accès aux informations et aux ressources de l'écosystème de TIC de la ville. La communauté représentait initialement un espace de coopération pour aider les entrepreneurs à résoudre les problèmes liés à leur entreprise ou au développement de leur technologie.

Les trois entrepreneurs instigateurs de cette dynamique se sont vus rejoindre par un nombre croissant d'autres entrepreneurs pour participer aux rituels de rencontres informelles en fin de journée, autour d'un verre dans une boulangerie/brasserie du quartier de São Pedro à Belo Horizonte, au Brésil.

Aujourd'hui, le nom a dépassé les limites géographiques de la région et représente l'ensemble des startups de la région métropolitaine de Belo Horizonte. Dans cet espace de détente, les discussions sur leurs affaires en cours se déroulent librement.

SPV est devenue une communauté autogérée du domaine des TIC qui partagent l'objectif de créer et faire fonctionner des startups compétitives et créatives. Les entrepreneurs considèrent que la meilleure façon d'atteindre ces objectifs est en échangeant entre eux des informations, des connaissances et des expériences vécues.

Cette concentration d'entrepreneurs de startups et l'importance qu'elle représente dans l'écosystème d'innovation de la ville de Belo Horizonte nous offre une richesse empirique qui répond à la représentativité théorique de la recherche.

Ces caractéristiques de notre terrain de recherche nous ont semblé porteuses d'intérêt par rapport à notre problématique. Cependant, nous avons également souhaité nous assurer de sa représentativité théorique par rapport à la théorisation des CoP proposée par Wenger en 1998.

Nous avons alors cherché à approfondir la description de la CoP de SPV selon les critères théoriques :

- Le caractère informel et spontané : la CoP interorganisationnelle de SPV est un groupe auto-organisé. Mette en avant la dimension informelle de la communauté de pratique (BROWN et DUGUID, 1991), intégrant la gestion de connaissances des différentes organisations (WENGER, MCDERMOTT e SNYDER, 2002) les considèrent comme un dispositif managérial.

- L'engagement mutuel des membres est basé sur la capacité des membres de connecter intelligemment leurs compétences et connaissances avec celles des autres afin de créer du sens dans la CoP. Cela suppose l'aide mutuelle. Wenger (1998) considère plus important qu'être capable de répondre à l'ensemble des questions posées est « savoir aider » et « se faire aider ».

- Le référentiel commun révèle l'évolution des dynamiques sociales et montre une réorientation du domaine de savoir, du référentiel commun et de la capacité de production de la pratique des membres. La CoP se trouve en situation de saisir une opportunité pour mieux comprendre, anticiper et innover tout en réduisant le temps de développement de nouveaux produits et services dans le secteur de la TIC, notamment de par les relations de coopétition entre les membres de la communauté inter-organisationnelle.

Le contexte dans lequel émerge SPV, les dynamiques sociales des acteurs (entrepreneurs de startups) et les logiques de fonctionnement (l'engagement mutuel, la participation/réification et le répertoire partage) nous permet de " classer " San Pedro Valley comme une CoP inter-organisationnelle, comme un cas d'innovation.

Pour analyser l'évolution de cette communauté, nous avons utilisé différentes méthodes de collecte de données. 


\section{Les méthodes de collecte et d'analyse de données}

Dans cette recherche l'approche qualitative de type étude de cas en profondeur apparaît comme la démarche la plus adaptée afin d'appréhender les causalités multiples d'un même contexte (MILES et HUBERMAN, 2003).

Pour rassembler un riche matériau empirique sur SPV nous avons mené une campagne d'entretiens semi-directifs d'une durée 1 h30 environ chacun entre 2013 et 2016 auprès des 32 entrepreneurs de SPV (codes INT) et de 7 représentants d'institutions dans l'écosystème d'innovation local.

Ces entretiens, réalisés en deux moments, entre 2013-2014 et entre 2015-2016, ont portés sur l'histoire de la communauté, le rôle des membres et des institutions concernés par les dynamiques à l'œuvre, la perception de la communauté, les dynamiques de fonctionnement et de réification selon les théorisations de Wenger (1998) sur les CoP et d'Agterberg, Bart, Marleen et al. (2010) sur les NoP.

Nous avons également collecté d'autres données primaires par l'observation non participante lors de 28 rencontres organisées par la communauté et entités de l'écosystème organisés au sein de l'écosystème d'innovation de la ville de Belo Horizonte. La participation à ces évènements réalisés sur le terrain nous a permis de mieux appréhender le contexte de la recherche, de nous familiariser et s'adapter avec le " langage et rituels » des membres de SPV ainsi que de connaître les acteurs de la CoP. Dans le cadre de l'observation non participante, pendant les rencontres physiques auxquelles nous avons participé nous avons pu étudier les dynamiques d'interactions des membres de SPV.

Les données primaires ont également été obtenues par la méthode netnographique (KOZINETS, 2002). Cette « méthodologie de recherche qualitative adapte les techniques de recherche ethnographiques à l'étude des cultures et des communautés émergeant à travers les moyens de communication électroniques» (KOZINETS, 2002, p. 3). L'objectif de la netnographie menée sur la communauté en analyse dans cette recherche a été de comprendre en profondeur, et sur la durée, les comportements sociaux des groupes communautaires et des individus rattachés à SPV, mais aussi des individus associés et en relation avec la CoP dans l'écosystème d'innovation de la ville.

Nous avons participé à la vie communautaire par l'inscription dans les groupes, toutefois, nous ne sommes pas intervenus dans les groupes virtuels, nous avons seulement participé en tant qu'observateur externe afin d'étudier les environnements virtuels de SPV.

Au total nous avons accédé à un volume expressif de messages: 340 pages de conversations sur le groupe WhatsApp pendante le période de septembre de 2015 à mai de 2016 et 64.800 messages et plus de 517 fichiers ont été partagés depuis sa création en 2014 sur le groupe Slack. Nous avons créé des fiches d'observation pour sélectionnés les messages pour l'analyse selon la thématique des messages échangées.

Nous avons ainsi retenu les messages échangés concernant le thème de l'étude. Elles portent sur les discours recueillis à travers les échanges en ligne, les postages et mail-listes sur les groupes communautaires de SPV et des réseaux rattachés.

Cette diversité d'instruments vise à optimiser nos chances de capter l'évolution de la réalité sociale de la CoP ainsi que le phénomène de coopétition à l'œuvre dans le temps, de façon longitudinale.

Pour analyser ce matériau nous avons procéder par triangulation par codage en un dictionnaire de thèmes (STRAUSS et CORBIN, 2004). Le codage repose sur un processus en deux étapes conforme suggéré par Miles et Huberman (2003) : le codage ouvert dans la phase exploratoire et le codage thématique dans la phase intensive.

Nos objectifs d'analyse ont été multiples : i) cerner le fonctionnement et l'évolution de SPV notamment par rapport aux interactions entre ces membres, à la gestion des événements et de la pratique, les dynamiques d'innovation ; ii) capter la perception des membres et du territoire, iii) détailler l'ensemble de transformations ayant eu lieu dans le réseau et plus généralement dans la ville de Belo Horizonte.

Les grandes catégories de thèmes structurent l'étude de cas présentés à suivre.

\section{L'analyse et les Résultats}

Le cas est structuré selon les trois ambitions affichées dans cette recherche : le lien entre CoP et NoP, les effets des NoP sur le territoire et l'évolution de la pratique de ces réseaux selon le processus de participation/réification. 


\section{L'évolution de San Pedro Valley: le passage d'une CoP à un NoP}

La structure de fonctionnement de la CoP de SPV est marquée par l'hétérogénéité des membres et par son caractère spontané, émergent, informel et d'autogestion.

À l'origine, la CoP s'appuie sur le besoin d'un petit groupe d'entrepreneurs de startups avec pour objectif d'améliorer le partage des connaissances entre eux. Les témoignages des membres présents depuis l'origine, indique le caractère spontanée, émergent et sans intervention du processus de création :

"San Pedro Valley est née sans prétention. En fait, c'était une blague qui a fini par être sérieuse. Mais commençons par le début : [...] » (INT4)

Les échanges informels de la CoP avaient lieu dans un café du quartier São Pedro et avaient des échanges informels sur leurs savoir-faire, leurs connaissances, leurs expériences, leurs difficultés, leurs succès et échecs. Les rencontres sans agenda et sans calendrier sont devenues un rendez-vous fréquent des entrepreneurs, pour partager et se détendre.

Le " lancement officiel » de la CoP a eu lieu fin 2011. Lors d'une de ces rencontres, l'un des entrepreneurs a fait une blague en faisant allusion aux nombreux entrepreneurs présents dans la région :

"Il a dit cette phrase qui allait rester dans le temps: Wow, ici on dirait la Silicon Valley, chaque jour nous rencontrons un CEO diune startup. Ici c'est le San Pedro Valley (faisant référence au quartier) ». (INT17)

Ainsi, cette rencontre informelle est considéré comme "l'événement fondateur " de la CoP de SPV, dans le sens où les membres se sont engagés pour faire des choses ensemble, comme l'exprime l'entrepreneur de l'INT28 : "SPV a été, entre guillemets, officiellement créée ».

Les entrepreneurs participent à ses interactions partageant " des problématiques de la manque de ressources et de soutient des entités du secteur » et " d'une passion pour le sujet de l'innovation ». Ainsi, ce groupe approfondit leurs savoirs et leurs expertises dans le domaine de management des startups en entretenant des interactions informels autour de leurs intérêts communs, mais aussi de leurs intérêts individuels. Cependant l'intérêt communautaire a priorité sur l'intérêt individuel.

Ainsi, les premières relations inter-organisationnelles des entrepreneurs de startup de SPV résultent notamment de la nécessité des entrepreneurs d'améliorer leurs processus d'innovation, d'avoir accès à des marchés, et tient également à la réduction des coûts pour l'innovation de leurs projets d'innovation. Le témoignage de l'entrepreneur INT6 illustre cet argument :

"La San Pedro Valley apparaît comme une recherche de solutions à nos problèmes. La phase de démarrage d'une startup est bien compliquée car normalement l'entrepreneur souffre du manque de ressources et de compétences. Et donc, à partir de ces rencontres, de l'échange de connaissances dans la communauté, des groupes qui s'aident commencent à se former » (INT6)

Le groupe n'a pourtant pas une réelle homogénéité. Chaque membre est propriétaire d'une entreprise avec sa propre organisation et ses propres spécificités :

"Le SPV sont des startups, des startups différentes [...]. Vous voyez ici une entreprise à l’avant et une derrière. Chacune avec ses particularités. C'est cette hétérogénéité qui fait que lsinformation soit fluide et qu'elle aide à réduire la courbe dıapprentissage de toutes les startups » (INT3)

Les résultants des premiers cas de succès des startups membres de la SPV sont un des facteurs déterminants pour la croissance et pour la visibilité de la CoP envers les autres startups pas encore membres mais aussi envers les entités du secteur de TI et d'autres acteurs de l'écosystème d'innovation (les universités, les investisseur, la presse). Par conséquent, le nombre de startups se propage à d'autres quartiers de Belo Horizonte.

Les échanges informels entre membres, face-à-face et de façon dyadique, évoluent vers la création et le développement de mécanismes d'intégration des connaissances dispersées entre les membres de façon à mieux gérer la dynamique du réseau.

Sans local propre, le groupe communique avec plus d'intensité à travers des outils technologiques électroniques : le site web de la communauté, leur page sur Facebook, ou, par le biais de groupes de discussions dans des outils de communication). 
Des communautés de pratique aux réseaux de pratique: une étude de l'évolution d'une communauté des startups de la ville de Belo Horizonte
Tatiane Barleto Canizela Guimarães | Luciana Castro Gonçalves Gláucia Maria Vasconcellos Vale

Ainsi, de plus en plus l'échange d'informations et le partage des connaissances se fait virtuellement sans atteindre au caractère informel et spontanée du réseau. Ces outils de communications permettent d'assurer la coordination du réseau (encastrement organisationnel) et d'aider les membres à comprendre son fonctionnement (encastrement social). Les membres peuvent également exploiter plus facilement les ressources disponibles selon les intérêts de chacun (encastrement de la pratique).

"Il existe un site web et donc l'entrepreneur qui souhaite faire partie de la communauté accède au site et remplit un formulaire dsintérêt. Parfois, il y a quelque membres de la communauté qui regardent et examinent si le gars est vraiment un entrepreneur de startup, ou si cela nıa rien à voir avec notre secteur. C'est vraiment un groupe très informel, très spontané » (INT3).

Les relations préexistantes à SPV (certains étaient des collègues à l'université ou disposaient des relations d'amitiés personnelles) marquent les dynamiques de relations entre les premiers membres de la CoP.

Des symboles, tel que le pain confectionné par les membres où le slogan « Made in San PedroValley! » est imprimé, sont des marques de ce rattachement identitaire.

La décontraction est aussi une caractéristique de l'identité des entreprises membres et de la CoP de SPV. Les espaces de travail sont irrévérencieux, presque une « marque de fabrique » des startups de la CoP.

Nous observons une motivation intrinsèque liée à la communauté chez la plupart des entrepreneurs interrogés. La CoP est source d'inspiration et de fierté pour les membres. Les conquêtes de chaque startup sont divulguées et commémorés par les autres membres, soit sur les réseaux sociaux (Twitter, LinkedIn, Facebook) soit sur les outils de communication de la CoP.

Le partage de connaissances peut avoir lieu entre startups à différents niveaux de maturité, entre collègues, partenaires, mais aussi entre concurrents au sein de SPV. Cette hétérogénéité de membres est cependant bien perçue par les membres de la communauté (encastrement social). Il s'agit d'un moyen pour créer de nouvelles ressources, d'apprendre le savoir-faire de son concurrent tout en protégeant son cœur de compétence et tout en enrichissant et élargissant le réseau (PREVOT, 2007). Par ailleurs c'est l'intérêt collectif qui prime. Il s'agit également de renforcer la légitimité de SPV dans la région.

Les membres insistent sur le fait que à l'origine SPV est « une communauté sans hiérarchie » (INT12) et « organique » (INT3).

"Malgré la proximité de plusieurs fondateurs, une formalisation ne ferait que prenez notre temps précieux, au lieu dıaller travailler dans nous startups, aller prendre soin diune institution. Cela nıa aucun sens. » (INT4)

Pour " gérer la CoP ", les activités sont partagées entre les leaders, les membres appartenant au noyau dur et qui ont participé depuis le début de la communauté et d'autres membres qui sont bien intégrés à la pratique. Ainsi, les entrepreneurs sont responsables par des activités spécifiques telles que représenter la CoP face aux entités de l'écosystème de la ville, répondre aux emails dirigés à SPV, participer à des interviews dirigés à la presse, gérer la page internet et modérer les outils de communications.

Qu'il s'agisse des échanges informels entre un groupe de 3 entrepreneurs au café du quartier ou des échanges pour le développement d'autres startups, la force du groupe est constaté par le nombre de participants qui a rapidement augmenté. Le groupe a progressivement évolué pour organiser des rencontres dans d'autres locaux, faisant émerger d'autres formes d'échanges plus formels tels les évènements organisés en partenariats avec les entités de l'écosystème (Seed, Sebrae, les universités et le gouvernement).

À mesure que les startups évoluent et que d'autres entrepreneurs s'intègrent aux dynamiques de la CoP, les membres essayent de créer une structure, une image et une mission à SPV. Les membres ont ainsi créé une marque et un slogan.

Le contexte interorganisationnel de la CoP présente un avantage concurrentielle pour les membres qui partagent et échangent leurs informations, leurs ressources et leurs compétences dans le but de surmonter leurs manque de ressources.

Actuellement, la CoP regroupe plus de 300 membres. Le numéro est significatif par rapport à la population de 2,5 millions d'habitants et aux données du secteur des startups présentées initialement.

Le réseau de coopération entre les startups passe par des relations informelles et formelles établies dans et à partir de la CoP. Ce réseau devient crucial pour la compétitivité des startups et exerce une influence positive pour la légitimité de la CoP dans le contexte d'innovation de Belo Horizonte. 


\section{Les effets de SPV sur le territoire : la légitimité du NoP et l'étendue de la pratique}

Les dynamiques sociales au sein de la CoP se nourrissent des relations sociales et économiques entre les entreprises. Un exemple est le fait d'un groupe d'entrepreneurs du noyau dur de SPV coopérer pour la réalisation d'un recensement de la réalité des startups dans le but de montrer leur pouvoir sur le marché des TIC de la ville et de s'imposer sur l'écosystème local. Le recensement a obtenu les données de 54 startups concernant l'ancienneté, le nombre d'employés et le nombre de postes de travail créés, le résultat comptable, les difficultés rencontrées par les entreprises dans la phase actuelle et les suggestions sur la façon dont les institutions publiques pourraient aider les entreprises.

Nos analyses montrent que la CoP devient une unité importante dans l'écosystème d'innovation de la ville en ce qu'elle :

- Incite à la création de nouvelles startups ;

- Véhicule et légitime le rôle des startups dans l'écosystème d'innovation ;

- Améliore le réseau des relations entre startups;

- Accroitre la visibilité des startups locales dans la ville et dans le pays.

En s'appuyant sur son succès, SPV a progressivement élargie son centre d'intérêt. Un groupe de membres incitent en effet les autres membres à développer des innovations plus responsables qui intègrent la dimension de respect de l'environnement, la dimension sociale par le soutien de populations en situation précaire, au-delà des retombées économiques apportées par le succès du réseau.

Autre exemple est illustré par un groupe des membres de SPV que se mobilisent pour participer à l'organisation dans cours d'entrepreneuriat offert aux élèves d'informatique de l'Université Fédérale de l'État de Minas Gerais. Les membres de la CoP ont participé à la restructuration de la discipline, ont donnés des cours portant sur des thématiques spécifiques selon leurs expertises et ont aussi participés en tant que « mentor » pour les élèves.

Nos analyses identifiant d'autant plus, un intérêt particulier des institutions de l'écosystème et du gouvernement local pour cette CoP en raison de leur visibilité et de la puissance des startups dans l'économie de la ville en raison du numéro des nouvelles entreprises et d'emplois créé.

Cet intérêt s'est manifesté principalement par le développement des programmes d'accélération des startups à Belo Horizonte. Les membres ont participés activement à la création du programme d'accélération de startups du gouvernement de l'État de Minas Gerais, le Seed en 2013. Cette action reflet l'approximation de l'État avec les startups de la ville.

Enfin, le rapprochement de la Fédération de l'Industrie au Brésil pour soutenir les startups à un stade avancé. Le programme FIEMG Lab crée en 2016 est une outre preuve de l'impact de SPV dans l'écosystème d'innovation de la ville.

La mobilisation de l'écosystème d'innovation favorise la visibilité et des partenariats institutionnels. SPV passe à s'imposer dans l'écosystème d'innovation et participer aux décisions en tant qu'une « entité » représentant des startups.

\section{Lévolution de la CoP selon trois processus de participation/réification distincts}

Par ailleurs, nous identifions trois phases distinctes qui marquent le développement progressif d'un groupe à une CoP interorganisationnelle et finalement à un NoP :

- La phase d'émergence des relations entre startups - Lors de l'émergence du groupe, en 2011, où la pratique était fondée sur l'échange très spontanée d'expériences vers la résolution de problèmes liés aux TIC et à la gestion des startups. Cette participation a donné lieux à la transformation de connaissances tacites en connaissances explicites.

- La phase de développement des relations vers une CoP - Devenue une CoP, les dynamiques sociales se sont renforcées. L'engagement mutuel a dépassé le partage de connaissances pour se fonder sur l'échange de ressources plus tangibles. Cette participation a dépassé le niveau des échanges interindividuels pour devenir des échanges interentreprises. En effet, ce mode de participation a donné lieu à une réification sous forme de " services gracieux » échangés entre startups membres, dans l'objective de répondre à des besoins stratégiques concernant l'innovation et la productivité à travers la création des programmes de partenariats entre les startups de la CoP.

- La phase de changement de la nature des relations vers un NoP - En atteignant une masse critique, la CoP devient progressivement un NoP grand et disperse géographiquement. Les membres bénéficient clairement au niveau économique de l'apprentissage collectif et de la croissance de son capital cognitif. Progressivement, au-delà de la 
création/développement des innovations, les membres de SPV affichent une volonté de développer la pratique de l'innovation responsable et assume un fort engagement social et politique dans la ville.

Mais naturellement, la logique de coopération n'est pas stable à travers le temps. Des signes de compétition peuvent être observés. Pour gérer ces contradictions, les entrepreneurs doivent échanger en permanence des signaux sur leur volonté de continuer à adopter un comportement coopératif, tout en concilient les logiques de coopération et de compétition entre membres.

Les résultats montrent ainsi une réorientation du domaine de savoir, du référentiel commun et de la capacité de production de la pratique.

La CoP e le territoire coevoluent selon des cycles de participation/réification de la pratique. Ce processus d'ouverture de la pratique en contexte d'innovation et ces effets sur le territoire sont exprimés par le développement du processus coopératif. L'accroissement du capital cognitif commun contribue à rendre l'échange de signaux de moins en moins coûteux.

L'existence de la CoP entre les entrepreneurs de SPV a joué un rôle spécifique dans l'extension vers un NoP. Les membres ont la culture de partage et association de ces contacts directs (de la participation) et les pratiques formalisées (de réification) du NoP. L'existence de la CoP de SPV a permis de consolider un réseau social reliant les individus et un processus d'apprentissage collectif reconnu. Appartenir à SPV est une source de fierté tout en donnant une légitimité dans l'écosystème qui ignorait sa présence avant son succès.

En raison de cette proximité auprès des acteurs de l'écosystème local, SPV se trouve en situation de saisir une opportunité pour mieux comprendre, anticiper et innover tout en réduisant le temps de développement de nouveaux produits et services dans le secteur de la TIC notamment de par les relations de la coopétition entre les membres.

\section{CONCLUSION}

L'analyse du cas de SPV a montré comment sont créées les relations communautaires inter-organisationnelles face à l'absence de structures de soutien au développement de startups et à l'innovation dans le territoire. Les liens solidaires forts entre les entrepreneurs émergent alors comme une innovation institutionnelle mettant en évidence une nouvelle dynamique des rapports sociaux face à la nouveauté dans la forme organisationnelle et relationnelle particulière (NORTH, 1997).

Le passage des échanges de connaissances au niveau interindividuels, puis de ressources au niveau inter-entreprises et enfin l'extension de la pratique en rapport avec le développement du territoire représentent trois modes distincts de participation/ réification qui marquent la co-évolution entre le réseau informel d'individus, l'innovation et le territoire. Cette recherche répond ainsi à l'appel de la littérature pour mieux étudier l'évolution des CoP dans le temps (DIBIAGGIO et FERRARY, 2003).

Cette recherche montre également que ce processus évolutif se présente selon un continuum entre CoP et NoP selon un mouvement d'ouverture à la participation à de nouveaux membres au sein du réseau, à d'autres acteurs de l'écosystème d'innovation et à la société civile du territoire.

Le renforcement de la légitimation permet d'en assurer la coordination et de s'imposer comme acteur majeur du développement entrepreneurial de l'innovation avec un fort engagement organisationnel et politique dans l'écosystème local.

Aujourd'hui, la CoP représente un canal de communication reconnu où circulent des connaissances, des récits d'expérience, des ressources et des actions de soutien entre membres.

Dans ce contexte étendu, certains membres plus engagés appartenant au noyau dur de la CoP jouent un roule déterminent dans la préservation de la confiance et de l'engagement inter-organisationnel entre membres malgré l'ouverture et la croissance vers le territoire.

Des travaux ultérieurs pourraient atténuer les limites de la présente recherche, notamment en s'intéressant davantage aux effets de cette dynamique communautaire évolutive sur le développement des innovations (en termes de créativité, de rythme de développement, de partenariats, de protection, etc.). Dans une perspective empirique, se pose également l'enjeu d'évaluer le rôle des CoP et des NoP afin d'évaluer leur dynamique économique dans l'écosystème d'innovation local. 
Des communautés de pratique aux réseaux de pratique: une étude de l'évolution d'une communauté des startups de la ville de Belo Horizonte
Tatiane Barleto Canizela Guimarães | Luciana Castro Gonçalves Gláucia Maria Vasconcellos Vale

\section{RÉFÉRENCES}

ACATE TECH REPORT. Panorama de inovação e tecnologia de SC. Florianópolis: Acate, 2015.

AGARWAL, N.; AGARWAL, R. Why Communities of Practice (CoP) are 'still' relevant for the organizations? Studies in Asian Social Science, v. 3, n. 2, 2016.

AGTERBERG, M. et al. Keeping the wheels turning: the dynamics of managing networks of practice. Journal of Management Studies, v. 47 , n. 1, p. 85-108, 2010.

BERENDS, H.; VAN BURG, E.; VAN RAAIJ, E. M. Contacts and contracts: cross-level network dynamics in the development of an aircraft material. Organization Science, v. 22, n. 4, p. 940-960, 2011.

BROWN, J. S.; DUGUID, P. Organizational learning and communitiesof-practice: toward a unified view of working, learning, and innovation. Organization science, v. 2, n. 1, p. 40-57, 1991.

CASTRO GONÇALVES, L. Strategizing across boundaries: revisiting knowledge brokering activities in French innovation clusters. Journal of Knowledge Management, v. 19, n. 5, p. 1048-1068, 2015.

COHENDET, P.; CRÉPLET, F.; DUPOUËT, O. Innovation organisationnelle, communautés de pratique et communautés épistémiques: le cas de Linux. Revue française de gestion, n. 5, p. 99-121, 2003.

COHENDET, P.; GRANDADAM, D.; SIMON, L. The anatomy of the creative city. Industry and innovation, v. 17, n. 1, p. 91-111, 2010.

COHENDET, P. et al. Epistemic communities, localization and the dynamics of knowledge creation. Journal of economic geography, v. 14, n. 5, p. 929-954, 2014.

DAMERON, S.; JOSSERAND, E. Le développement d'une communauté de pratique. Revue française de gestion, n. 5, p. 131-148, 2007.

DIBIAGGIO, L; FERRARY, M. Communautés de pratique et réseaux sociaux dans la dynamique de fonctionnement des clusters de hautes technologies. Revue d'économie industrielle, v. 103, n. 1, p. 111-130, 2003.

FICHTER, K., BEUCKER, S. Managing innovation communities: best practice reports. In: FICHTER, K.; BEUCKER, S. (Eds.). Innovation communities. Berlin: Springer, 2012. p. 227-267. Disponible en: < $\underline{\text { http:// }}$ dx.doi.org/10.1007/978-3-642-22128-6 4>. Accès à: 14 sept. 2018.

FIGUEIREDO, P. N.; PIANA J. When "one thing (almost) leads to another: a micro-level exploration of learning linkages in Brazil's mining industry. Resources Policy, v. 49, p. 405-414, 2016.

GHERARDI, S. To start practice theorizing anew: the contribution of the concepts of agencement and formativeness. Organization, v. 1, n. 19, p. 1-19, 2015.

GHAURI, P. N.; GR ØNHAUG, K. Research methods in business studies: a practical guide. New Jersey: Financial Times Prentice Hall, 2005.

GOGLIO-PRIMARD, K. Cracking the network boundaries: How Networks of Practice and Communities of Practice contribute to knowledge sharing in ICT sector. In: ANNUAL IMP-CONFERENCE, 30., Bordeaux, Proceedings... Bordeaux: IMP, 2014.
GONGLA, P.; RIZZUTO, C. R. Evolving communities of practice: IBM Global Services experience. IBM systems journal, v. 40, n. 4, p. 842862, 2001.

HYSLOP, K. Open innovation activities and network competence as drivers of SME performance. In: ISPIM INNOVATION CONFERENCE, 27., 2016, Porto. Proceedings... Porto: SPIM, 2016. p. 1.

LEVERING, R. et al. Continuity and change in interorganizacional project practices: the dutch shipbuilding industry, 1950-2010. International Journal of Project Management, v. 31, n. 5, p. 735-747, 2013.

LEVINA, N.; VAAST, E. The emergence of boundary spanning competence in practice: implications for implementation and use of information systems. MIS Quarterly, v. 29, n. 2, p. 335-363, 2005.

JENSEN, M. B. et al. Forms of knowledge and modes of innovation. In: LUNDVALL, B-A. The Learning Economy and the Economics of Hope. London: Anthem Press, 2016. p. 155.

JOSSERAND, E. The network organization: the experience of leading French multinationals. Cheltenham: Edward Elgar, 2004.

JOYAL, A. Les APL au Brésil: une adaptation opportune des SPL à la française? Géographie, économie, société, v. 10, n. 2, p. 185-197, 2008.

KOZINETS, R.V. The field behind the screen: using netnography for marketing research in online communities. Journal of marketing research, v. 39, n. 1, p. 61-72, 2002.

LAVE, J.; WENGER, E. Communities of practice. Retrieved June, v. 9, p. 2008, 1998.

LUNDVALL, B. A. (Ed.). National Systems of Innovation: Towards a Theory of Innovation and Interactive Learning. London: Printer, 1992.

LESSER, E. L.; STORCK, J. Communities of practice and organizational performance. IBM systems journal, v. 40, n. 4, p. 831-841, 2001.

MCDERMOTT, R. Community Development as a Natural Step Like all living things, communities of practice have a natural life cycle. The key to starting, developing and sustaining them is understanding and resolving the natural tensions of each stage of development. Knowledge Management Review, v. 3, p. 16-19, 2000.

MILES, M. B.; HUBERMAN, A. M. Analyse des données qualitatives. De Boeck Supérieur, 2003.

MOINGEON, B. et al. Inter-organizational communities of practice: Specificities and stakes. Les Cahier de Recherche, v. 857, p. 18, 2006.

NORTH, D. C. Understanding economic change. In: NELSON, J.; TILLY, C.; WALKER, L. Transforming post-communist political economies. Washington: National Academy Press, 1997. p. 13-18.

PATTINSON, S.; PREECE, D. Communities of practice, knowledge acquisition and innovation: a case-study of science-based SMEs. Journal of Knowledge Management, v. 18, n. 1, p. 107-120, 2014.

STRAUSS, A.; CORBIN, J. Les fondements de la recherche qualitative. Techniques et procédures de développement de la théorie enracinée. Fribourg: Academic Press, 2004.

VAAST, E. O brother, where are thou? From communities to networks of practice through intranet use. Management Communication Quarterly, v. 18, n. 1, p. 5-44, 2004. 
Des communautés de pratique aux réseaux de pratique: une étude de l'évolution d'une communauté des startups de la ville de Belo Horizonte
Tatiane Barleto Canizela Guimarães | Luciana Castro Gonçalves Gláucia Maria Vasconcellos Vale
YIN, R. K. Applications of case study research second edition. Applied Social Research Methods Series, v. 34, 2002.

WENGER, E. Communities of practice: learning, meaning, and identity. Cambridge university press, 1998.
WENGER, E; MCDERMOTT, R. A; SNYDER, William. Cultivating communities of practice: a guide to managing knowledge. Harvard Business Press, 2002.

Tatiane Barleto Canizela Guimarães

Doctorat à I'Université Paris-Est,ESIEE Paris et I'Université PUC Minas; Professeur au Centro Universitário Unibh et Newton Paiva, Belo Horizonte, MG

- Brésil. Email: tbarleto@gmail.com

Luciana Castro Gonçalves

Doctorat à I’Ecole Centrale Paris; Professeur Associé, ESIEE Paris, IRG/Université Paris-Est, Paris, France. Email: Iuciana.castro@esiee.fr

Glaucia Maria Vasconcellos Vale

Post-Doctorat à I'Université de Paris IV- Sorbonne; Doctorat à I'Université Federal de Lavras; Professeur PUC Minas, Belo Horizonte, MG - Brésil. Email: galvale@terra.com.br 